\title{
ESTUDO DAS PROPRIEDADES HIDRODINÂMICAS DA FORMAÇÃO SERGI NUMA ÁREA EXPERIMENTAL NA REGIÃO DE COCOROBÓ-BAHIA
}

\author{
OLIVAR A. L. DE LIMA* e CARLOS A. DIAS*
}

\begin{abstract}
Studies have been conducted for determination of detailed lithological characteristics of a sequence of Sergi sandstones from data colected during the drilling of six wells, in the Cocorobó region, in the Northeast of Bahia. Hydraulic parameters have been determined by laboratory tests on sandstone cores and samples from the drilling, showing quite uniform values, which averaged $3,1 \times 10^{-3} \mathrm{~cm} / \mathrm{s}$ for the permeability and $29 \%$ for the effective porosity, and presenting anisotropy about $40 \%$ greater for the horizontal permeability. The laboratory tests included granulometric and petrographic analysis and X-ray difratometry, and have shown that the Sergi Formation for the area studied is quite uniform, little cemented, and has only a small amount of clays and fine silt (less than $5 \%$ ). A well test of the saturated lower section of the sandstones permitted to estimate a value of the permeability somehow lower compared to the laboratory one, and equal to $0,7 \times 10^{-3} \mathrm{~cm} / \mathrm{s}$. This value is neverthless consistent with the laboratory results and the electrical well log, which evidenciate an increase in the clay content towards the basis of the sandstone sequence. A combination of these two results yielded the better values, given as $K=2,5 \times 10^{-3} \mathrm{~cm} / \mathrm{s}$ and $\eta_{\mathrm{c}}=29 \%$. The values of these parameters constitute the basic knowledge for defining the operation conditions for the adduction pipeline and injection system, which will be necessary to be constructed for a saturation experiment with the Cocorobo reservoir, in order to evaluate the feasibility of storage of water artificially in underground in that semi-arid region.
\end{abstract}

INTRODUÇÃo Na região de Cocorobó, situada no Município de Euclides da Cunha, no extremo norte do Estado da Bahia, foi selecionado um sítio experimental, compreendendo $3,1 \mathrm{~km}^{2}$ de superficie, para a investigação da possibilidade da injeção e do armazenamento artificial de água, em reservatórios subterrâneos sedimentares, subutilizados nas condições naturais do ambiente semi-árido brasileiro. $O$ reservatório seleciónado é constituído por uma seqüência de arenitos da Formação Sergi com espessura média de $45 \mathrm{~m}$.

O mapeamento geológico e os estudos geofisicos realizados mostraram que a Formação Sergi ocorre na região, intercalada entre litologias argilosas, numa estrutura em blocos, limitada por falhas de gravidade (Lima, 1979). Um destes blocos constitui o reservatório experimental, cuja configuração geométrica superficial vem mostrada na Fig. 1. Este bloco foi selecionado em vista de suas características favoráveis, determinadas com base em observações geológicas de superficie e na uniformidade de seu comportamento elétrico em resposta a sondagens de resistividade (Lima, 1979).

Este trabalho apresenta os estudos de campo e de laboratório conduzidos para determinar os coeficientes hidráulicos do reservatório, bem como para detalhar suas características litológicas. Tais resultados são úteis na previsão do comportamento do reservatório em resposta à injeção através de poços e na definição das condições de operação do sistema de tra tamento e adução, a ser ali implantado (Lima, 1979).

PERFURAÇÃO E PERFILAGEM ELÉTRICA DE POÇOS Duas perfurações de testemunhagem com sonda Winkie, P-1 e P-2, separadas entre si de $500 \mathrm{~m}$, ao longo da direção do acamamento litológico, foram executadas na área teste de Cocorobó (Fig. 1). Em P-1, com $52 \mathrm{~m}$ de profundidade, foram perfurados aproximadamente $45 \mathrm{~m}$ da Formação Sergi e obtidos cerca de $22 \mathrm{~m}$ de testemunhos, a maioria com coroa e barrilete de $60,3 \mathrm{~mm}$ de diâmetro (BX). Em P-2, com $23 \mathrm{~m}$ de profundidade, foram atravessados $21 \mathrm{~m}$ da Formação Sergi, tendo sido recuperados cerca de $12 \mathrm{~m}$ de testemunhos com coroa e barrilete de $49,2 \mathrm{~mm}$ de diâmetro (AX). Na Fig. 2, estão mostrados os perfis litológicos dos furos, as zonas de recuperação, a descrição macroscópica do material perfurado, e os valores de permeabilidade medidos no laboratório.

Foram perfurados ainda, com sonda rotativa, três poços piezométricos, $\mathrm{Pz}_{\mathrm{z}-1}, \mathrm{P}_{\mathrm{z}}-2$ e $\mathrm{Pz}-3$, e um poço pioneiro, $\mathrm{PN}-1$, convenientemente localizados

* Programa de Pesquisa e Pós-Graduação em Geofísica/Instituto de Geociências, Universidade Federal da Bahia. 40.000 Salvador, Bahia, Brasil 

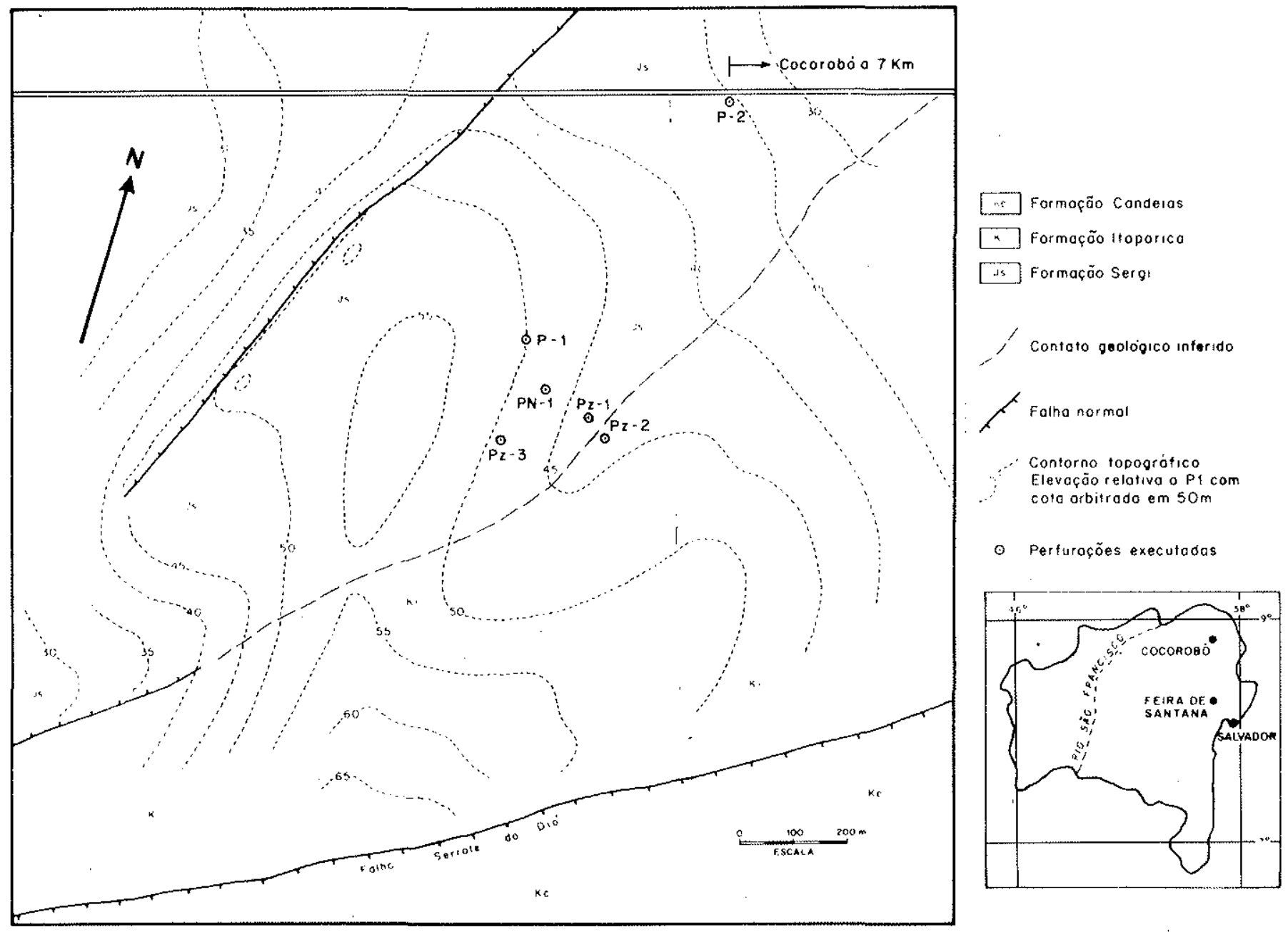

Figura 1 - Mapa Geológico e Topográfico da área teste de Cocorobó, mostrando a localização das perfurações

na região de experimentação, conforme mostrado na Fig. 1. Para cada poço, amostras de calha foram obtidas a intervalos de um metro de perfuração.

Os poços piezométricos foram perfurados com diâmetro de 6 polegadas, atravessando toda a espessura da Formação Sergi e revestidos com tubos plásticos PVC de 2,5 polegadas de diâmetro, ranhurados nas zonas permeáveis. Na definição das zonas de colocação dos tubos ranhurados foram usadas ainda indicações adicionais das perfilagens elétricas dos furos. Estas perfilagens de poço forneceram também informaçôes qualitativas sobre as características litológicas da Formação Sergi. As profundidades finais de perfuração de $\mathrm{Pz}-1, \mathrm{Pz}-2$ e $\mathrm{Pz}-3$ foram de $80 \mathrm{~m}, 90 \mathrm{~m}$ e $72 \mathrm{~m}$, respectivamente.

$\mathrm{O}$ poço pioneiro $\mathrm{PN}-1$ foi perfurado até à base da Formação Sergi com diâmetro de 18 polegadas, revestido com tubos e filtros de 12 polegadas de diâ" metro até à profundidade de $50 \mathrm{~m}$, e com tubos e filtros de 8 polegadas de diâmetro até à profundidade final de $70 \mathrm{~m}$, conforme esquematizado na Fig. 3 . PN-1 deverá ser usado na fase de experimentação como poço de injeção.
O equipamento utilizado na perfilagem elétrica dos furos foi o sistema da Gearhart-Owen Inc. com o módulo ELM-202, para fornecer medidas simultâneas da resistividade aparente para dois dispositivos normais, e do potencial espontâneo como função da profundidade.

A Fig. 4 representa os perfis litológicos dos furos $\mathrm{Pz}-2$ e $\mathrm{Pz}-3$, e os resultados de suas perfilagens elétricas. Embora os poços Pz-1 e PN-1 não disponham de perfilagens elétricas, os resultados podem ser extrapolados para os mesmos com base na análise das amostras de calha. Devido ao elevado contraste de resistividade entre as camadas de folhelhos e aquelas de arenitos, as curvas de resistividade aparente permitem estabelecer, com precisão, a posição dos contatos entre as mesmas. Verifica-se também que as camadas de arenito de granulação mais fina são caracterizadas por valores mais baixos de resistividade aparente, em consequiência de uma maior quantidade de argila disseminada, conforme foi observado nas amostras de calha coletadas naqueles poços. Nas zonas mais permeáveis do arenito observa-se, nos perfis elétricos, que houve invasão do filtrado da lama de perfuração, 


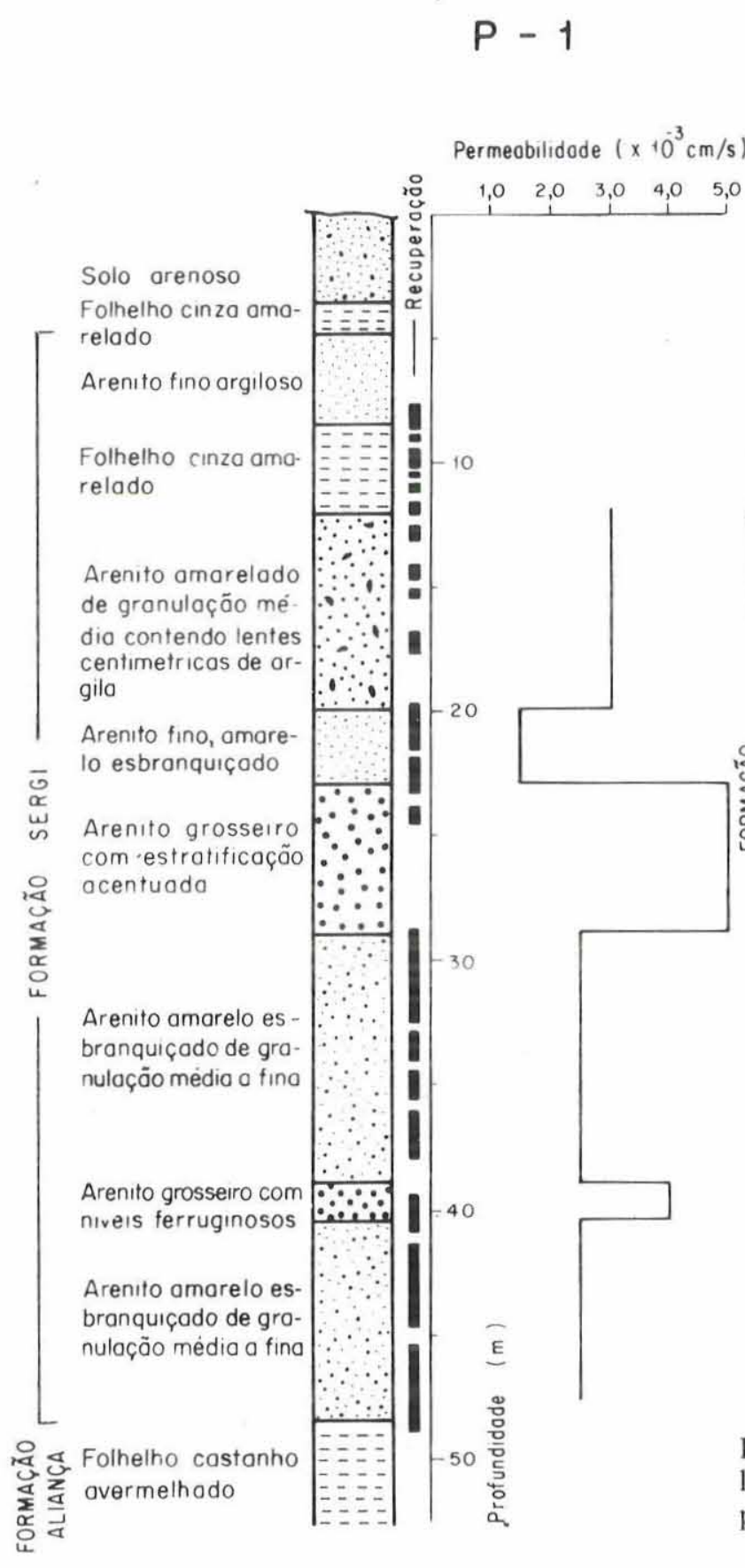

conforme indicado pela resistividade progressivamente mais baixa com o aumento do raio de investigação normal-curto comparado com o normal-longo. A curva do potencial espontâneo em $\mathrm{Pz}-2$ registra uma pequena anomalia de voltagem na região dos arenitos. Abaixo da profundidade de $60 \mathrm{~m}$, o retorno da curva do potencial espontâneo a um valor próximo da linha de base do folhelho, associado com a diminuição do valor da resistividade aparente de cerca de $300 \Omega \mathrm{m}$, para aproximadamente $100 \Omega \mathrm{m}$, sugere fortemente um aumento no teor de argila na base da formação. Em Pz-3, logo abaixo de $20 \mathrm{~m}$ de profundidade, a curva de potencial espontâneo apresenta uma deflexão para baixo, indicando a interface entre o are-
$P-2$

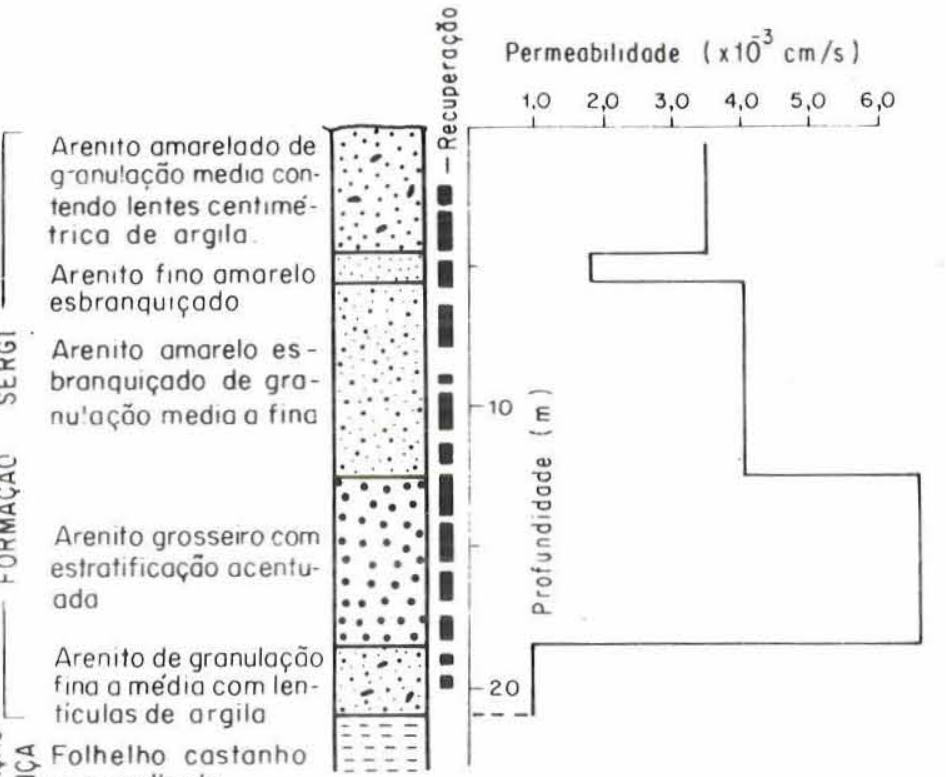

Figura 2 - Perfis litológicos, distribuição da amostragem e valores de permeabilidade vertical contra profundidade, nos poços testemunhados P-1 e P-2 nito de granulação fina, que possui intercalações de argila no topo da formação, e a faixa de arenitos puros que se estende até cerca, de $60 \mathrm{~m}$ de profundidade. Novamente, a uma profundidade ligeiramente maior do que $60 \mathrm{~m}$, a deflexão em sentido contrário da curva de potencial espontâneo, associada com a diminuição acentuada da resistividade aparente para um valor próximo de $100 \Omega \mathrm{m}$, relaciona-se a um aumento no teor de argila no arenito, na base da Formação Sergi.

Esses resultados indicam que a Formação Sergi, na área de experimentação, é caracterizada por uma espessa seção de arenito de permeabilidade relativamente elevada, conforme inferido da diminuição da 


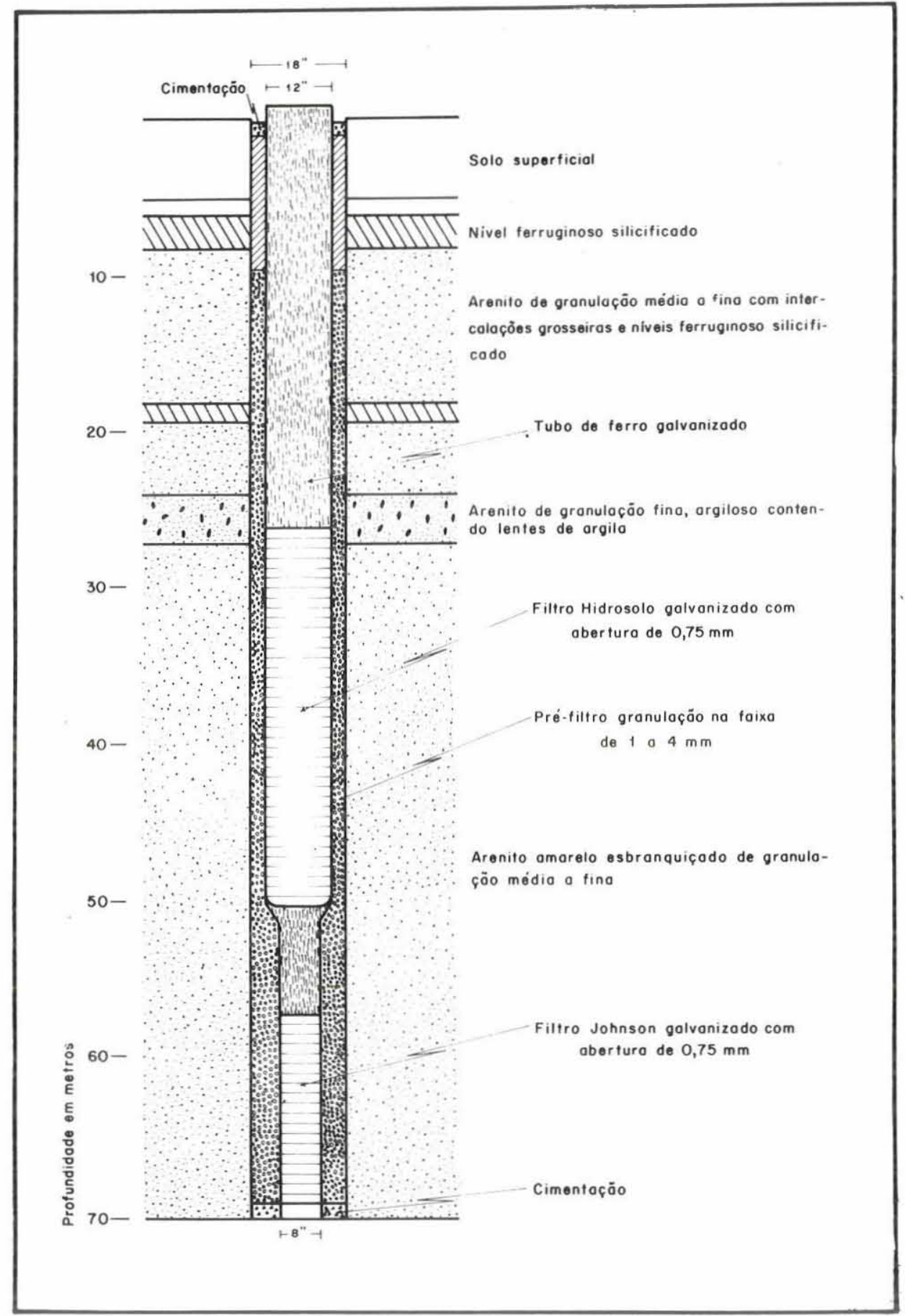

Figura 3 - Detalhes e especificação de construção do poço principal PN-1

resistividade aparente com o aumento do raio de investigação para os dois dispositivos normais utilizados, e do decaimento do valor do potencial espontâneo. Verifica-se, também, a presença de uma seção inferior menos permeável e, provavelmente, um pouco mais argilosa, conforme se interpreta dos valores de potencial espontâneo e da resistividade aparente nos perfis, abaixo de $60 \mathrm{~m}$ de profundidade.
INVESTIGAÇÃO DE LABORATÓRIO Procedimentos operacionais Foram selecionados os testemunhos obtidos em P-1 e P-2 e 85 amostras cilíndricas, confeccionadas com cerca de $38,5 \mathrm{~mm}$ de comprimento. Por causa do pequeno mergulho da Formação Sergi no sítio experimental de Cocorobó, as amostras foram consideradas como aproximadamente orientadas na direção perpendicular à estratificação do 


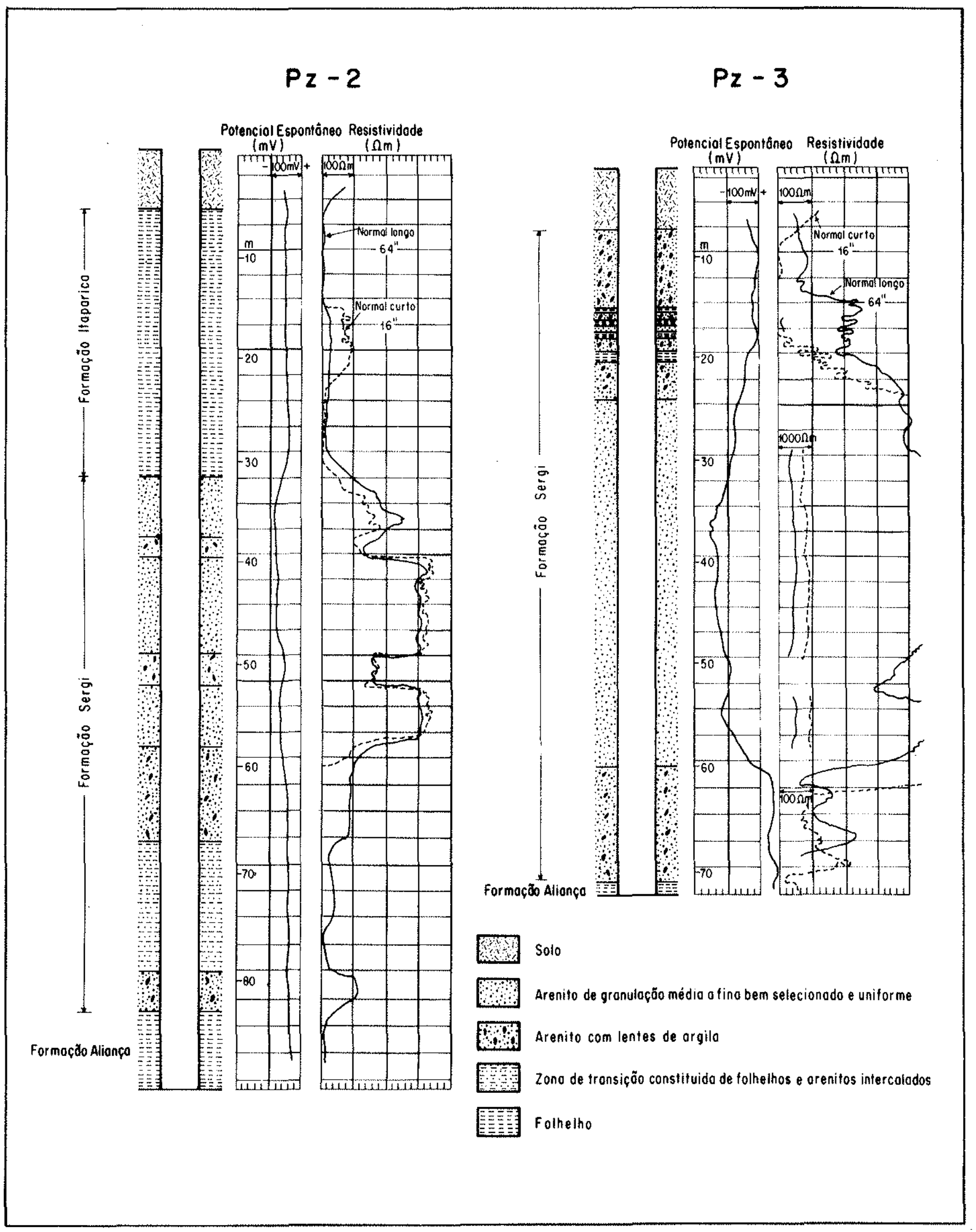

Figura 4 - Seções litológicas e perfilagens elétricas dos poços piezométricos Pz-2 e PZ:3 
arenito. Para verificar efeitos de anisotropia, 20 testemunhos de maior diâmetro, obtidos em P-1, foram cortados em cubos de cerca de $26 \mathrm{~mm}$ de aresta, de faces paralelas e faces perpendiculares à estratificação.

Sobre 65 frações de testemunhos, das quais 40 diretamente associadas às amostras cilíndricas, foram realizadas determinações granulométricas. Em face das dificuldades para obtenção de amostras representativas com perfuratriz rotativa, apenas 10 amostras de calha, cuidadosamente selecionadas de cada poço de observação, e 20 amostras do poço pioneiro foram usadas para análise granulométrica.

A determinação da porosidade foi feita pelo método das pesagens (Belikov et al, 1967), utilizando uma balança técnica de precisão com erro inferior a 0,001 grama. As amostras foram lavadas com água destilada para eliminação de impurezas e posteriormente secas em estufa durante 12 horas, a uma temperatura entre $60^{\circ}$ e $100^{\circ} \mathrm{C}$. Em seguida, as amostras foram pesadas no ar e, subsequientemente, saturadas com água destilada, sob ação de uma bomba de vácuo durante 12 horas. As amostras saturadas foram novamente pesadas no ar e na água. $O$ cálculo da porosidade foi feito usando a expressão:

$$
\eta_{\mathrm{s}}=\frac{P_{\mathrm{s}}-P}{P_{\mathrm{s}}-P_{\mathrm{l}}} \times 100 \%
$$

onde $\eta_{\mathrm{c}}$ é a porosidade efetiva, definida como a relação em $\%$ do volume de poros interconectados para o volume total da amostra, $P_{s}$ o peso da amostra saturada, $P$ o peso da amostra seca e $P$, o peso da amostra saturada imersa no líquido de saturação.

$\mathrm{Na}$ determinação da permeabilidade foi utilizado um permeâmetro de líquido Ruska n. ${ }^{\circ} 1013$, que funciona sob altura piezométrica constante. Neste instrumento, as medidas de permeabilidade são obtidas determinando o tempo necessário para que uma certa quantidade de liquido, a uma temperatura conhecida, passe através da amostra sob um gradiente de pressão prefixado. A permeabilidade é calculada pela expressão:

$$
k=\frac{\mu V L}{A p t}
$$

onde $k$ é a permeabilidade intrínseca da amostra (em darcys), $\mu$ a viscosidade dinâmica do líquido na temperatura da experimentação (em centipoise), $V$ o volume de liquido que flui através da amostra $\left(\mathrm{em} \mathrm{cm}^{3}\right)$, $t$ o intervalo de tempo para que o volume fixado do líquido flua através da amostra (em segundos), $p$ a diferença de pressão aplicada na amostra (em atm), $L$ o comprimento da amostra $(\mathrm{em} \mathrm{cm})$, e $A$ a área de sua seção transversal $\left(\mathrm{em} \mathrm{cm}^{2}\right)$. $\mathrm{O}$ comprimento e diâmetro de cada amostra foi medido com precisão de $0,1 \mathrm{~mm}$. A permeabilidade ou condutividade hidráulica $K, \mathrm{em} \mathrm{cm} / \mathrm{s}$, é obtida para a água a temperatura de $20^{\circ} \mathrm{C}$, multiplicando os valores de $k$ determinados em darcys pelo fator $0,9613 \times 10^{-3} \mathrm{~cm}^{-1} \mathrm{~s}^{-1}$, igual a $\gamma / \mu$, onde $\gamma$ é o peso específico do líquido. Neste trabalho, adotaremos a unidade $\mathrm{cm} / \mathrm{s}$, por conveniência.

Foram obtidas medidas de permeabilidade em cada amostra, inclusive com o cuidado de inverter a amostra. Uma vez que os resultados foram quase coincidentes, foram adotados os seus valores médios.

RESULTADOS EXPERIMENTAIS A Fig. 5 ilustra curvas de distribuição granulométrica típicas das zonas areníticas da Formação Sergi, na região estudada. Verifica-se, desta figura, que os arenitos são bem uniformes, conquanto ocorra variação na dimensão média dos grãos. $\mathrm{O}$ diâmetro efetivo dos grãos $\left(d_{10}\right)$, um parâmetro definido pela dimensão na qual ocorre $10 \%$ em peso de grãos com diâmetros inferiores a esse valor, é na média das amostras de $0,184 \mathrm{~mm}$, com desvio padrão de $0,04 \mathrm{~mm}$. O coeficiente de uniformidade $(U)$, definido pela razão $d_{60} / d_{10}$, varia de 1,2 a 2,2. Em todas as amostras analisadas, o teor médio de argila e silte fino (diâmetro inferior a $0,031 \mathrm{~mm}$ ) é inferior a $5 \%$. Análises espectrográficas por difratometria de raios- $X$, mostraram que as argilas disseminadas são do grupo da caolinita.

A porosidade efetiva dos arenitos varia de $23 \%$ a $34 \%$, com um valor médio para 58 determinaçóes em amostras de $\mathrm{P}-1$ de $29,1 \%$ e desvio padrão de $1,57 \%$. A média de 25 determinaçôes em amostras de P-2 foi de $28,9 \%$ com desvio padrão de $3,25 \%$.

Os valores do coeficiente de permeabilidade vertical para os poços analisados variaram de aproximadamente $6,4 \times 10^{-4} \mathrm{~cm} / \mathrm{s}$, para um diâmetro efetivo de $0,11 \mathrm{~mm}$ e 1,6 para o coeficiente de uniformidade, até aproximadamente $5,3 \times 10^{-13} \mathrm{~cm} / \mathrm{s}$ a um diâmetro efetivo de $0,27 \mathrm{~mm}$ e 2,0 para o valor de $\mathrm{U}$. O valor médio das amostras de P-1 foi de $2,46 \times 10^{-3}$ $\mathrm{cm} / \mathrm{s}$ com desvio padrão de $1,57 \times 10^{-3} \mathrm{~cm} / \mathrm{s}$. As amostras de P-2 forneceram o valor médio de 4,08 $\times$ $\times 10^{-3} \mathrm{~cm} / \mathrm{s} \mathrm{com}$ desvio padrão de $1,78 \times 10^{-3} \mathrm{~cm} / \mathrm{s}$. Todavia, devido a variações laterais de espessura nas camadas de arenitos, um valor mais representativo do comportamento médio da permeabilidade da formação pode ser obtida calculando para cada poço a soma das transmissividades de cada zona de arenito e dividindo o resultado pela espessura total dos arenitos. Procedendo dessa maneira, obtém-se os valores de $2,78 \times 10^{-3} \mathrm{~cm} / \mathrm{s}$ e $3,94 \times 10^{-3} \mathrm{~cm} / \mathrm{s}$ em P-1 e P-2, respectivamente. Na Fig. 2, vem mostrado também o gráfico da variação da permeabilidade em termos das profundidades das camadas.

Um ajuste pelo método dos mínimos quadrados da relação entre coeficientes de permeabilidade vertical e diâmetro eficaz para o conjunto de 40 amostras dos dois poços, fornece a relação empírica:

$$
K=72,8\left(d_{10}\right)^{182}
$$

com um coeficiente de correlação de 0,912 (Fig. 6). Esta expressão permite estimar valores de permeabilidade em $10^{-3} \mathrm{~cm} / \mathrm{s}$, quando o parâmetro $d_{\mathrm{i} 0}$ vem 


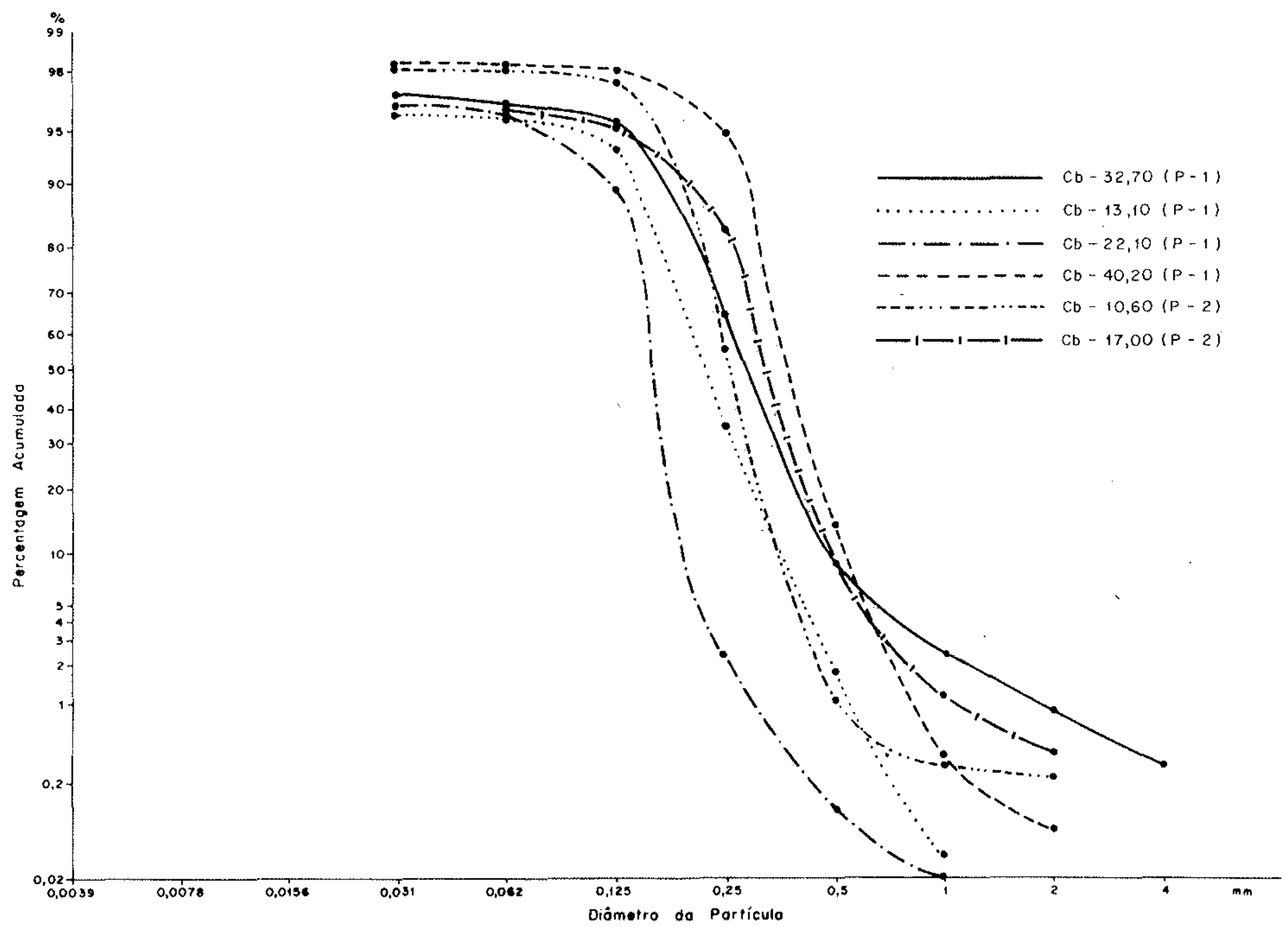

Figura 5 - Distribuição granulométrica em amostras típicas dos arenitos da Formação Sergi. O número da amostra corresponde à sua profundidade no furo

dado em $\mathrm{mm}$, nos locais dos poços piezométricos e do pioneiro, a partir da análise granulométrica das amostras coletadas em calha, durante a perfuração daqueles poços. Notando que a permeabilidade intrínseca $k$ é uma propriedade somente do meio poroso e, nas condições do fluxo lamelar, proporcional ao quadrado do valor médio do diâmetro dos poros (BEAR, 1972), bem como que $K$ é proporcional a $k$ por um fator que só depende do fluido $(\gamma / \mu)$, verifica-se que a relação (3) possui um elevado grau de coerência, e que o parâmetro $d_{10}$ deve ser da ordem de grandeza do diâmetro médio dos poros do arenito Sergi, na área estudada.

Somente em amostras de P-1 foi possível cortar amostras cúbicas para determinações de permeabilidade perpendicular e paralela a estratificação dos arenitos. Os resultados de 20 determinações desse tipo mostram que os arenitos possuem anisotropia de aproximadamente $40 \%$. O valor médio para a permeabilidade medida na direção perpendicular foi de
$2,38 \times 10^{-13} \mathrm{~cm} / \mathrm{s}$ com desvio padrão de $1,5 \times 10^{-3}$ $\mathrm{cm} / \mathrm{s}$, enquanto para a direção paralela obteve-se $3,26 \times 10^{-3} \mathrm{~cm} / \mathrm{s}$ com desvio padrão de $1,8 \times 10^{-3}$ $\mathrm{cm} / \mathrm{s}$. Na Fig. 7, compara-se a linha de isotropia com a distribuição dos valores obtidos. Verifica-se que a permeabilidade na direção horizontal $\left(K_{\mathrm{x}}\right)$ é geralmente maior que a permeabilidade na direção vertical $\left(K_{z}\right)$. $\mathrm{O}$ ajuste pelo método dos mínimos quadrados fornece a relação:

$$
K_{\mathrm{x}}=1,68 K_{\mathrm{z}}^{0.80}
$$

com um coeficiente de correlação de 0,926 . A anisotropia de permeabilidade está provavelmente relacionada a orientação preferencial de minerais tabulares (micas, argilas, etc.), o que tende a favorecer o fluxo na direção paralela ao acamamento.

No que se refere aos poços piezométricos e ao pioneiro, foram cuidadosamente selecionadas amostras de calha para análise granulométrica. Para cada 


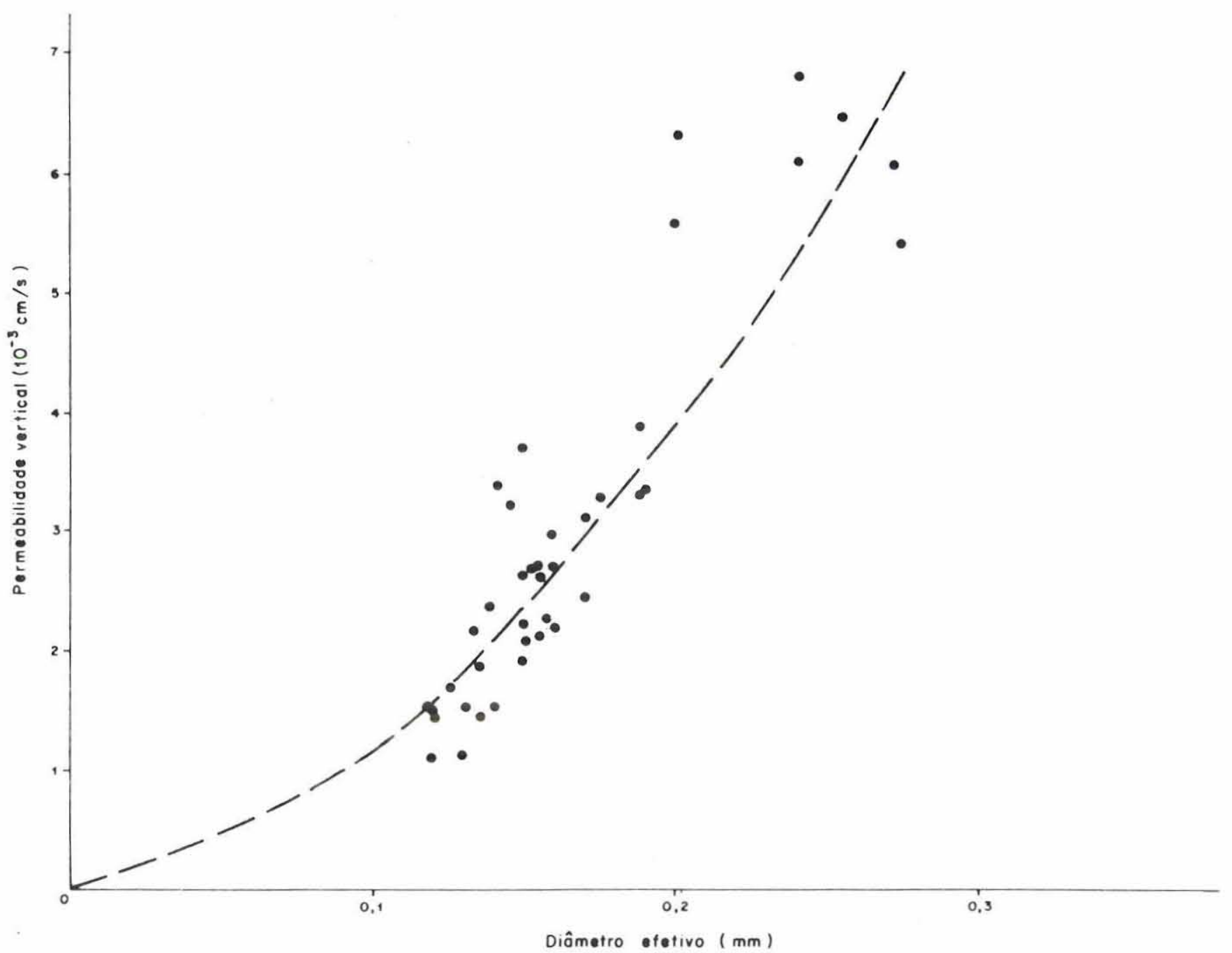

Figura 6 - Correlação entre a permeabilidade vertical e o diâmetro efetivo para amostras de arenito da Formação Sergi

amostra, calculou-se o diâmetro efetivo e o coeficiente de uniformidade. Usando a expressão (3) foram estimados valores da permeabilidade vertical para esses poços, conforme apresentados na Tab. 1. Os valores numéricos, assim estimados, são comparáveis àqueles obtidos em testemunhos, indicando uma certa regularidade litológica da formação, lateralmente, na área studada.

Tabela I - Valores médios dos parâmetros granulométricos e permeabilidade estimada nos poços piezométricos e principal. O número entre parêntesis se refere ao número de amostras

\begin{tabular}{c|c|c|c|c|c|c}
\hline \multirow{2}{*}{$\begin{array}{c}\text { Poços de observação } \\
\text { e poço principal }\end{array}$} & \multicolumn{2}{|c|}{ Diâmetro efetivo $(\mathrm{mm})$} & \multicolumn{2}{c}{$\begin{array}{c}\text { Coeficiente de } \\
\text { uniformidade }\end{array}$} & \multicolumn{2}{c}{ Permeabilidade $\left(10^{-3} \mathrm{~cm} / \mathrm{s}\right)$} \\
\hline & Média & $\begin{array}{c}\text { Desvio } \\
\text { padrão }\end{array}$ & Média & $\begin{array}{r}\text { Desvio } \\
\text { padrão }\end{array}$ & Média & $\begin{array}{c}\text { Desvio } \\
\text { padrão }\end{array}$ \\
& $0,182(10)$ & 0,05 & $2,9(10)$ & 0,8 & $3,32(10)$ & 1,9 \\
Pz-1 & $0,204(10)$ & 0,05 & $3,0(10)$ & 1,3 & $4,04(10)$ & 1,8 \\
Pz-2 & $0,171(10)$ & 0,04 & $3,0(15)$ & 0,9 & $2,98(10)$ & 1,4 \\
P P - & $0,170(20)$ & 0,04 & $2,8(20)$ & 0,6 & $3,11(20)$ & 1,6 \\
\hline
\end{tabular}




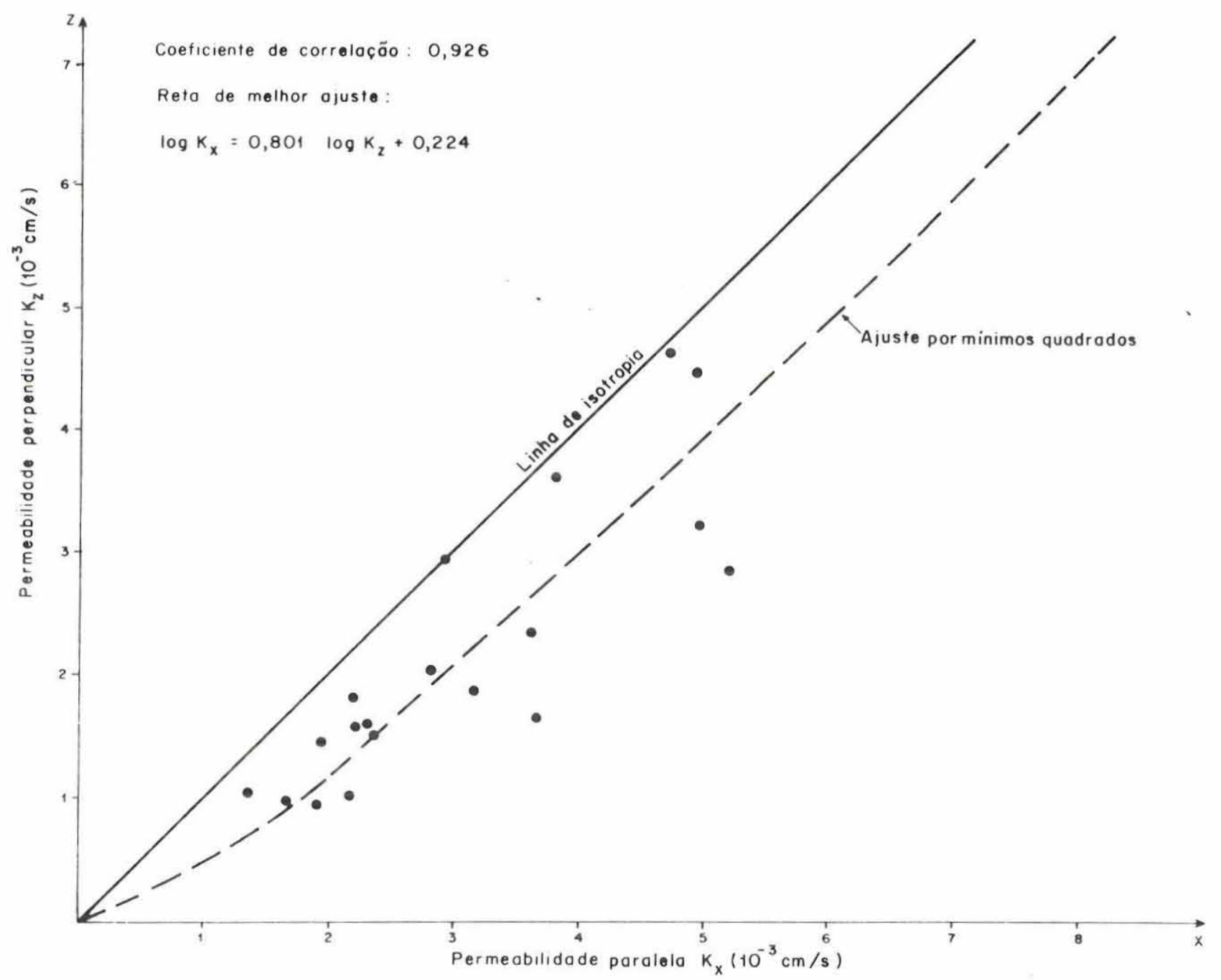

Figura 7 - Anisotropia de permeabilidade em 20 amostras de arenito da Formação Sergi

ESTIMATIVAS DA PERMEABILIDADE IN SITU A observação direta do nível hidrostático nos poços mostra que apenas uma fração basal correspondente a cerca de $7 \%$ da espessura do arenito está saturada de água nas vizinhanças de PN-1. Isto torna impraticável determinar in situ os parâmetros hidrodinâmi$\cos$ do arenito a partir de ensaios convencionais de bombeamento. Em tais condições, para estimar valores de permeabilidade in situ, no poço PN-1, foram realizados testes de recuperação natural e de injeção de curta duração, sob condições controladas.

Segundo Ferris et al. (1962) foi desenvolvido um procedimento para determinação do coeficiente de transmissividade, a partir da recuperação do nível de água num poço após a extração de água por baldeamento. Em qualquer ponto da curva de recuperação a seguinte equação é aplicável:

$$
s^{\prime}=\frac{V}{4 \pi T t\left(\exp \left(r_{\mathrm{p}}^{2} \eta_{\mathrm{e}} / 4 T t\right)\right)}
$$

onde $s^{\prime}$ é o rebaixamento residual, $V$ o volume de água removido durante o baldeamento do poço, $T$ o coeficiente de transmissividade, $\eta_{\mathrm{c}}$ a porosidade efetiva, $t$ o intervalo de tempo após o término do baldeamento, e $r_{\mathrm{p}}$ o raio efetivo do poço. Para grandes valores de $t$, o termo exponencial em (5) se aproxima da unidade, de modo que

$$
s^{\prime}=\frac{4}{4 \pi T t} .
$$

Após a remoção por baldeamento de um volume de $730 l$, a Fig. 8 ilustra a curva de recuperação do nível da água em PN-1, medida usando um dispositivo especialmente desenvolvido para esse fim (Lima, 1969). Verifica-se desta figura que, decorridos 300 minutos do término do baldeamento, o rebaixamento residual foi de $0,15 \mathrm{~m}$. Conhecendo a espessura saturada do aquífero na posição de equilíbrio, o uso da equação (6) permitiu estimar o valor de $0,8 \times 10^{-3}$ 


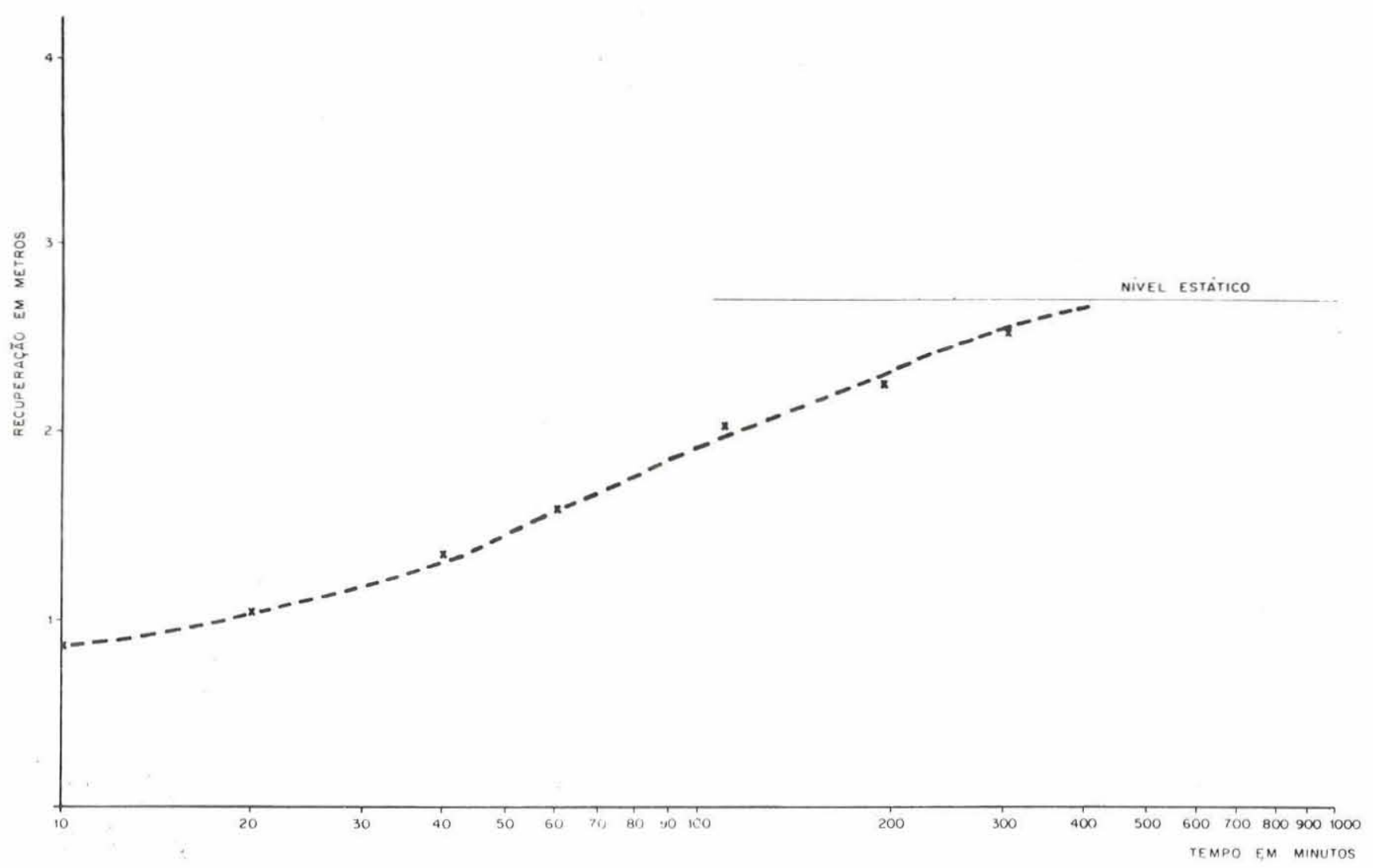

Figura 8 - Curva de recuperação do nível de água no poço principal PN-1, após a extração de 730 litros de água

$\mathrm{cm} / \mathrm{s}$ para o coeficiente de permeabilidade da seção basal saturada do arenito.

Um teste de injeção de curta duração, seguindo o procedimento empírico desenvolvido pelo United States Bureau of Reclamation, denominado de Teste B (Krynine e Judd, 1957), foi conduzido para verificação do valor estimado a partir do ensaio de recuperação. Este teste consiste basicamente em injeção de água no poço a uma vazão constante, num regime de fluxo estacionário, e determinação do coeficiente de permeabitidade usando a expressão (Krynine e Judd, 1957)

$$
K=\frac{2 Q}{r_{\mathrm{e}}\left(C_{\mathrm{s}}+4 r / r_{\mathrm{e}}\right)(T+H-A)}
$$

onde $Q$ é o valor da vazão, $r$ o raio externo do revestimento, $r_{\mathrm{e}}$ a relação da área aberta sobre a área total do filtro, $T$ a altura de água acima do nível estático original, $H$ a altura da água acima da base do filtro, $A$ o comprimento do segmento de filtro usado pela água, e $C_{\mathrm{s}}$ um coeficiente numérico que é obtido de um gráfico de $C_{\mathrm{s}}$ versus valores de $A / r_{\mathrm{e}}$ (Krynine e Judd, 1957).

No teste executado em PN-1, a água foi injetada abaixo do nível estático do poço através de um conduto, a uma vazão constante de $334 \mathrm{~cm}^{3} / \mathrm{s}$ utilizando uma motobomba Hypron, com motor de $5 \mathrm{HP}$ de potência. Um estado de equilibrio aproximado foi atingido após 180 minutos de injeção, quando o nível de água no poço situou-se à profundidade de $56,6 \mathrm{~m}$. Essa leitura do nível da água foi calibrada por observações contínuas durante cerca de 5 minutos. A variação do nível estático, nesse intervalo, foi inferior a $5 \%$. Conhecendo-se a profundidade de equilibrio e as especificações do poço, foram determinados os valores de $T, H, A, r_{\text {c }}$ e $C_{\text {s }}$ que substituidos em (7) forneceram o valor de $0,6 \times 10^{-3} \mathrm{~cm} / \mathrm{s}$ para a permeabilidade da seção basal do arenito Sergi, em torno do poço $\mathrm{PN}-1$.

Os valores de permeabilidade estimados a partir dos ensaios de campo estão situados entre $1 / 4$ e $1 / 5$ dos valores médios determinados com ensaios de laboratório sobre testemunhos, e estimados para PN-1 com base em análises granulométricas. Tais diferenças são atribuídas a um aumento no teor de argila na seção inferior da formação, provocando uma redução apreciável no comportamento médio da permeabilidade. Este fato foi diretamente observado nos testemunhos obtidos na base da formação do poço P-2 (Fig. 2), bem como foram inferidos pelos resultados das perfilagens elétricas nos poços (Fig. 6).

Associando os dados de laboratório aos dados de campo, é possível estimar um valor médio de per- 
meabilidade para o reservatório estudado, considerando o mesmo constituído de duas seções: a basal, ligeiramente mais argilosa, para a qual se atribui permeabilidade $0,7 \times 10^{-3} \mathrm{~cm} / \mathrm{s}$ de acordo com os dados de campo, e a superior com valor médio de $3,1 \times 10^{-3}$ $\mathrm{cm} / \mathrm{s}$, definido estritamente pelos dados de laboratório. Considerando as espessuras estabelecidas para essas duas seções, chega-se a um valor médio de aproximadamente $2,5 \times 10^{-3} \mathrm{~cm} / \mathrm{s}$. O valor real poderá ser um pouco maior que o estabelecido por esses procedimentos, em consequiência da presença de uma componente de fissura associada ao fraturamento do arenito, conforme observado nos dados de sísmica de refração (Lima, 1979).

CONCLUSÕES Os resultados das determinações de porosidade e permeabilidade dos arenitos da Formação Sergi, obtidos em laboratório, indicam que o reservatório é de permeabilidade anisotrópica e varia com os estratos. A correlação dos dados de permeabilidade, obtidos sobre testemunhos com os parâmetros de análises granulométricas dos poços P-1 e P-2, permitiram o estabelecimento de uma relação empírica entre permeabilidade e diâmetro efetivo, que tornou possível estimar valores de permeabilidade com base nos dados granulométricos obtidos de amostras de calha dos poços de observação e de injeção.

Embora existam questões quanto ao uso em problemas de campo de valores de permeabilidade obtidos em laboratório, muitos autores, Johnson e Greenkorn, 1960; Bredehoeft, 1964; Johnson e Sniegocki, 1967; Barker e Worthington, 1973) têm demonstrado uma boa concordância entre valores de permeabilidade obtidos em amostras no laboratório e valores determinados a partir de testes convencionais de bom- beamento. Neste trabalho, supõe-se que os dados de laboratório são representativos das condições de campo, e que o valor médio da permeabilidade, gerado pela combinação dos dados de campo com os dados de laboratório, e o valor médio da porosidade efetiva determinado no laboratório permitem caracterizar os parâmetros hidrodinâmicos do reservatório de Cocorobó, na escala do seu volume.

As análises granulométricas, petrográficas, e por difratometria de raios $\mathrm{X}$, demonstram que a Formação Sergi na área estudada é uniforme, pouco cimentada e contém um baixo teor de argila do grupo da caolinita. Por isso, espera-se não haver dificuldades na injeção e na recuperação da água injetada, por ser pouco provável a ocorrência de entumescimento provocado por colóides de argila.

Os poços de observação e o principal, perfurados na área de experimentação, foram completados tecnicamente usando informações de perfilagens elétricas e de amostragens regulares em calha, e construídos atendendo especificação de seus usos futuros na fase de injeção experimental no reservatório de Cocorobó. Os resultados das perfilagens elétricas serviram também para mostrar a uniformidade petrofisica do arenito.

As observações diretas do nível hidrostático, obtidas nos poços de observação e no principal indicam que o reservatório está subutilizado nas condições naturais do ambiente semi-árido.

Agradecimentos Os autores agradecem à Profa. Yeda de Andrade Ferreira, por suas valiosas críticas e estímulo, à Superintendência do Desenvolvimento do Nordeste (SUDENE), ao BNDE/FUNTEC, à FINEP e ao CNPq pelo financiamento a esta pesquisa.

\section{BIBLIOGRAFIA}

BARKER, R.D. e WORTHINGTON, F.F. - 1973 - Some hidrogeophysical properties of the Bunter Sandstone of Northwest England. Geoexploration, 2(3): 151-170.

BEAR, J. - 1972 - Dynamics of Fluids in Porous Media. America Elsevier, New York, $764 \mathrm{pp}$.

BELIKOV, B.P., ZALESSKII, B.V., ROZAVOV, Yu.A., SANINA, E.A. e TIMCHENKO, I.P. - 1967 - Method of Studying the Physico Mechanical Properties of Rocks. In: Physical and Mechanical Properties of Rocks. B.V. Zaleskii Ed: 1-58. Israel Program for Scientific Translation, Jerusalém.

BREDEHOEFT, J.D. - 1964 - Variation of permeability in the Tensleep Sandstone in the Bighorn Basin, Wyoming, as interpreted from core analysis and Geophysical logs. U.S. Geol. Survey, Prof. Paper n. 501 D: 166-170.

FERRIS, J.G., KNOWLES, D.B., BROWN, R.H. e STALLMAN, R.W. - 1962 - Theory of Aquifer Tests. U.S. Geol. Survey, Water-Supply Paper n. 1536-E, 173 pp.

JOHNSON, C.R. e GREENKORN, R.A. - 1960 - Comparison of core analysis and drawdown test results from a water bearing upper Pennsylvanian Sandstone of central Oklahoma (abst.). Geol. Soc. Am. Bull. 71(12): 1898.

KRININE, D.D. e JUDD, W.R. - 1957 - Principles of Engineering Geology and Geotechnics, McGraw-Hill, New York, 730 pp.

LIMA, O.A.L. de - 1979 - Estudo da Utilização de Reservatórios Subterrâneos Naturais para Armazenamento de Água em uma Área Experimental na Região Semi-Árida do Nordeste Brasileiro. Tese (Doutor em Geofísica), Universidade Federal da Bahia, Salvador, $145 \mathrm{pp}$ 